\title{
Analysis of Dialogue Stimulated by Science Videos and Reference Materials
}

\author{
Daichi Sunouchi \\ Tokai University, 4-1-1 \\ Kitakaname, Hiratuka, Knagawaga \\ 259-1292, Japan
}

Email: 3bef1120@mail.tokai-u,jpl

\author{
Kiyoshi Nosu \\ Tokai University, 4-1-1 Kitakaname, \\ Hiratuka, Knagawaga 259-1292, \\ Japan \\ Email: nosu@wing.ncc.u-tokai.ac.jp
}

\begin{abstract}
Recently, many have begun to believe that learning and training approaches known as learner-centered, active learning, and cooperative learning improve learning and practicing performance and are more effective than traditional lectures. Moreover, in addition to paper-based materials such as textbooks, face-to-face co-located communication frequently utilizes digital video and other visual reference materials. However, no previous studies have examined the precise faceto-face behavior of dialogue participants stimulated by video and other reference materials. Therefore, this paper describes the dialogue stimulated by science videos and reference materials based on data from $\mathbf{1 0}$ male university students measured while using first-, second-, and third-person view videos, as well as utterances recorded during the measurements.
\end{abstract}

\section{INTRODUCTION}

$\mathrm{T}$ EACHERS and instructors in traditional education and training are responsible for defining learning purposes and objective areas of learning tasks, and for designing and assessing learning and training processes. Recently, many have begun to believe that learning and training approaches referred to as learner-centered, active learning, and cooperative learning improve learning and practicing performance and are more effective than traditional lectures [1]-[4]. The concept of collaboration and cooperation includes allowing individuals to enrich their own experience of acquiring knowledge on their own accord.

An important factor enabling these learning approaches is Information and Communication Technology (ICT), which provides learners with many kinds of tools that can be used at schools, including preschools, and business workplaces to enhance the way in which they acquire diversified knowledge, skills, and experiences [4].

Collaboration through digital video-mediated communication has been widely used, and research comparing videomediated with face-to-face communication has been reported [4]-[6]. For example, O'Mally et al. compared the dialogue in video-mediated communication with that in faceto-face co-location communication. The results showed that both video-mediated and face-to-face speakers use visual cues to check for mutual understanding. They also suggested that speakers are less confident in their mutual understanding when they are not physically co-located.

Moreover, in addition to paper-based materials such as textbooks, face-to-face co-located communication frequently uses digital video and other visual reference materials. These previous studies have not examined the precise faceto-face behavior of dialogue participants stimulated by video and other reference materials.

Therefore, this paper describes the dialogue stimulated by science videos and reference materials based on data from 10 male university students measured while using first-, second-, and third-person view videos, as well as utterances recorded during the measurements.

\section{METHODS}

\section{A. Dialogue stimulation materials}

The following materials were prepared for the measurements:

(1) Video: "Lives of creatures on Earth"

Copyright-free Hi-Vision materials were obtained from the NHK Archives Video Library [7]. Copyright-free BGM materials were obtained from OVA-SYNDROME's FREE BGM website [8]. All videos of seven creatures were edited using Windows Movie Maker, Microsoft.

(2) Reference materials

The important parts of the video were captured and edited for printed reference materials, which are shown in Fig.1

\section{B. Participants}

The study participants were 10 male university engineering students in their early twenties.

\section{Video shooting views}

The first-person view video is that of a participant, and the second-person view video is that of their dialogue partner. The 
third-person view video is the viewpoint of a person other than the dialogue pair.

\section{Measurement procedures}

(a) Equipment and reference materials

- Wearable video camera for the first/second-person view (Panasonic HX-A500, Japan)

- Digital video camera for the third-person view (Panasonic HC-V360M)

- Personal computer for viewing an instructional origami skill video (Toshiba Dynabook D41, Japan)

- Reference materials: video on the lives of various creatures and video captures.

(b) Measurement setup and procedure

Fig. 1 shows the equipment setup. The behavior of the participants was recorded from different viewpoints. Their dialogue was also recorded using a voice recorder. The measurement procedure was as follows:

(1) A participant wore a wearable camera near his ear.

(2) Before watching the video (1 minute): After distributing printed materials and presenting the discussion theme, "Important creatures on Earth", the dialogue pair started a free discussion after watching the reference material, as shown in Fig.2. The participants did not receive any additional instructions or requests apart from having free discussions during the measurement.

(3) During the video viewing (4 minutes and 30 seconds): The participants had a free discussion on the lives of creatures they watched in the reference video and materials.

(4) After watching the video (3 minutes): The participants continued a free discussion on the lives of creatures on Earth. They re-watched the reference video and materials if necessary.

(5) After the dialogue was finished, the recorded utterances were transcribed verbatim. The text data were then used to analyze emotional changes during the time series.

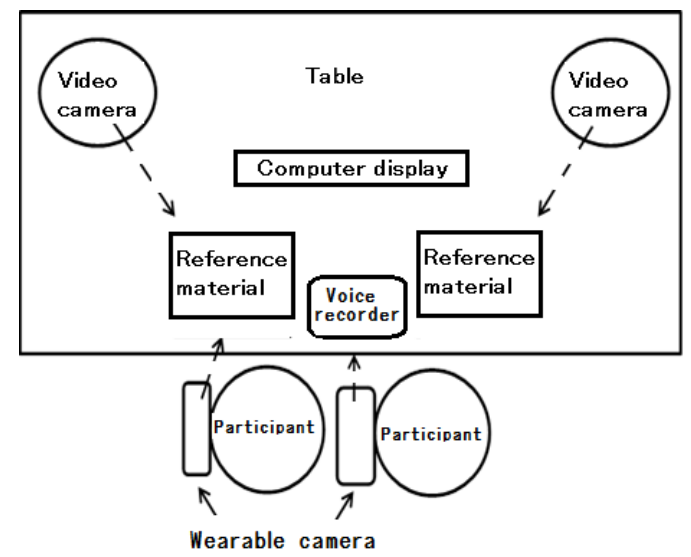

Fig.1 Video shooting arrangement (top-view)

\section{MEASURED RESULTS AND ANALYSIS}

Fig.2(a) - (e) shows characteristic behavior images (photos) of Pairs A, B, C, D, and E, respectively.

Fig. 3 shows time sequence changes in utterance intentions. The utterance intentions are classified as follows: Intention 1: Questions and proposals in the dialogue Intention 2: Agreement or disagreement between partners in the dialogue

Intention 3: Opinions regarding the video themes Intention 4: Others

Intention 4 of Pairs A and B increased, suggesting that they may reached consensus regarding the initial dialogue theme after watching the video.

\section{CONCLUSION}

The paper described the dialogue stimulated by science videos and reference materials using data from 10 male university students measured using first-, second-, and third-person view videos and utterances recorded during the measurements. Possible strategies for promoting efficient dialogue were described based on the results. The main results are summarized as follows.

(1) Watching videos and other reference materials stimulates face-to-face dialogue.

(2) Dialogue saturation is defined as (i) a decrease in the utterance frequency rate, (ii) an increase in dialogue intent or content that is not directly related to the initial theme.

(3) These behaviors are found regardless of how frequently the participant looks at the dialogue partner.

(4) Monitoring dialogue saturation factors (i) and (ii) in item (2) above could help realize computerized interventions and assistance that would enable face-to-face dialogue and discussions to become fruitful and efficient.

Since (i) and (ii) can be detected by speech recognition and emotion estimation by physical expressions [9], in the future, in conjunction with AI and voice synthesis, computerized interventions and assistance for efficient dialogue could be realized by suggesting dialogue or development themes [10],[11] for further constructive discussion if the dialogue becomes saturated.

To realize this, further investigations on the measurements, analysis and assessments of different dialogue themes by different category subjects are needed so that emerging technologies for learning and training are fully utilized in practice. 


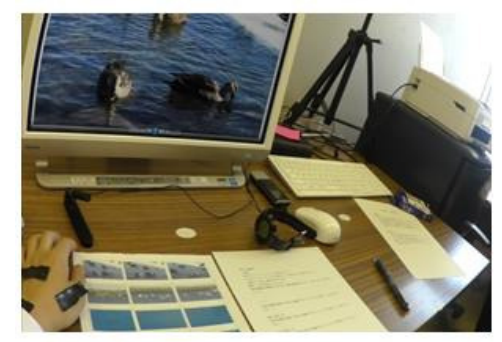

Participant I's first-person view (Participant II's second-person view)

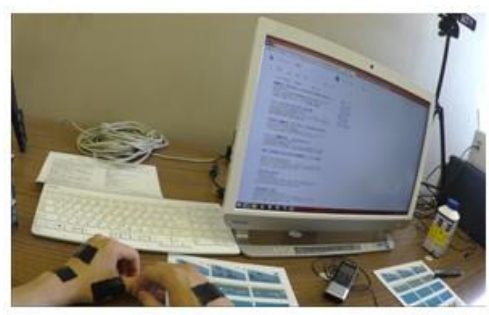

Participant III's first-person view

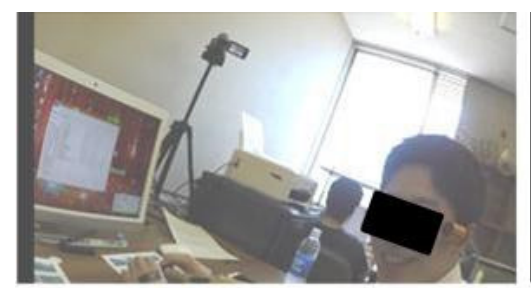

Participant V's first-person view

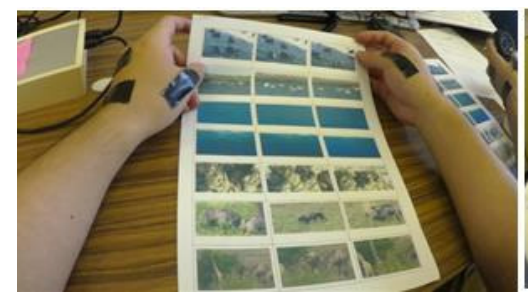

Participant VII's first-person view

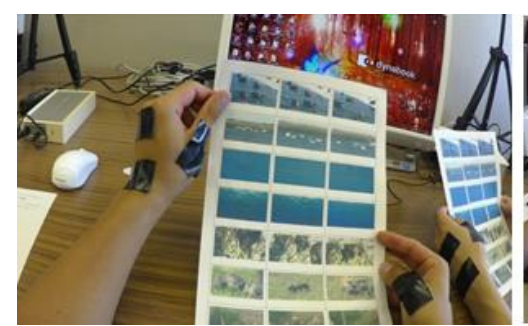

Participant IX's first-person view

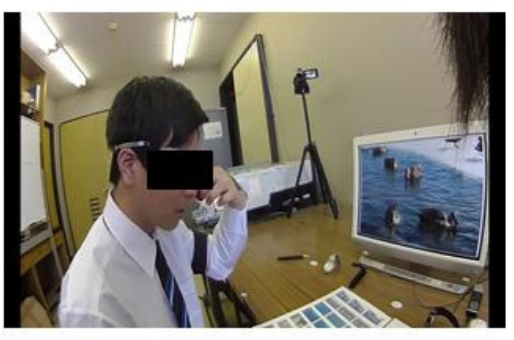

Participant II's first-person view (Participant I's second-person view)

(a)

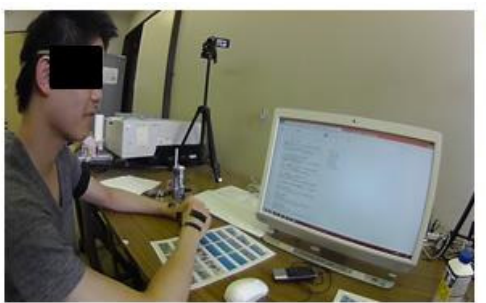

Participant IV's first-person view

(b)

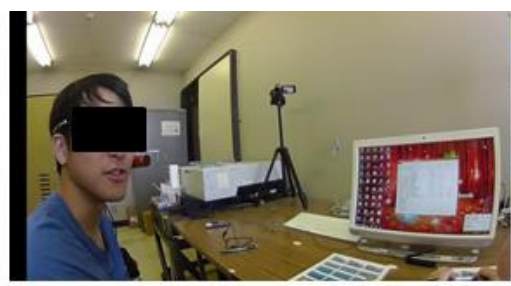

Participant VI's first-person view

(c)

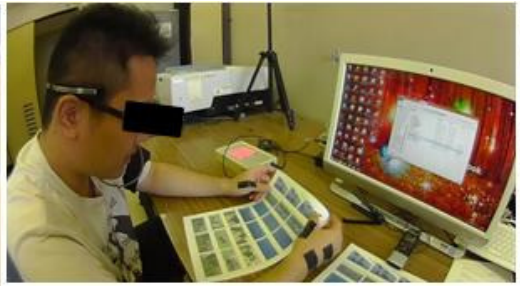

Participant VIII's first-person view

(d)

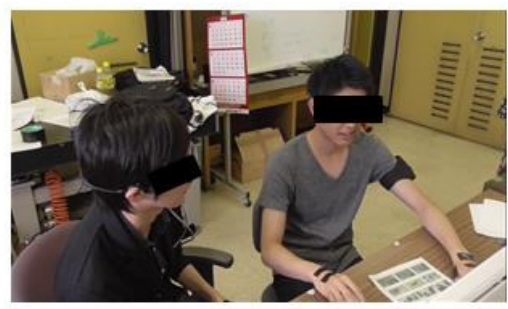

Participant III and IV's third-person view

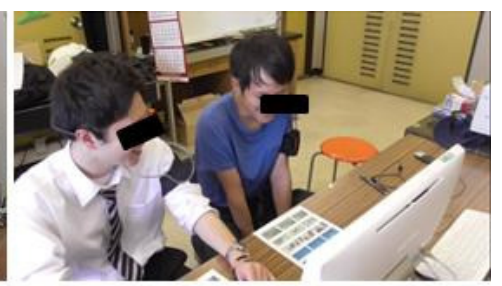

Participant V and VI's third-person view

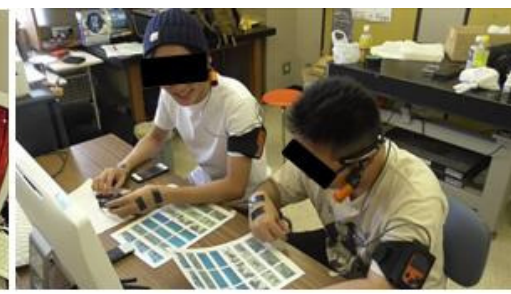

Participant VII and VIII's third-person view

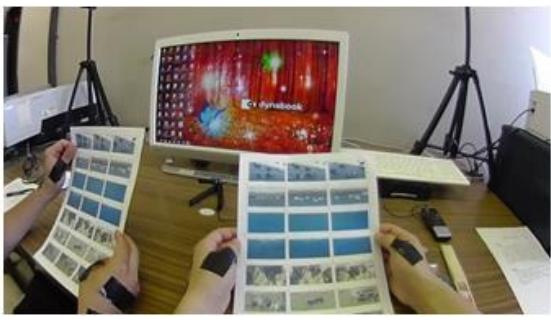

Participant X's first-person view

(e)

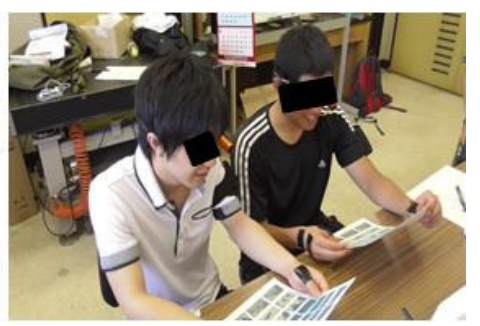

Participant IX and X's third-person view

Fig.2 Characteristic behavior images 


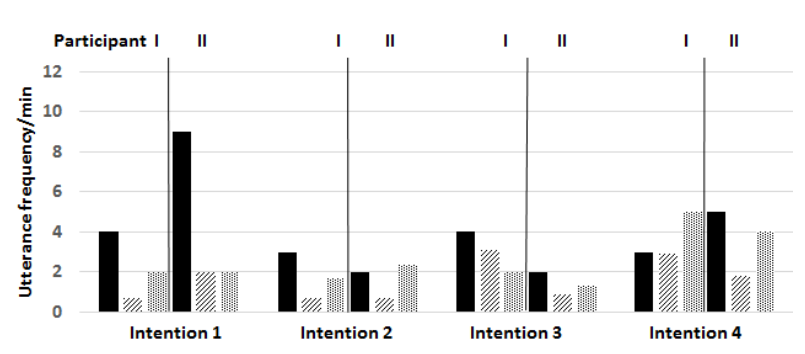

(a)

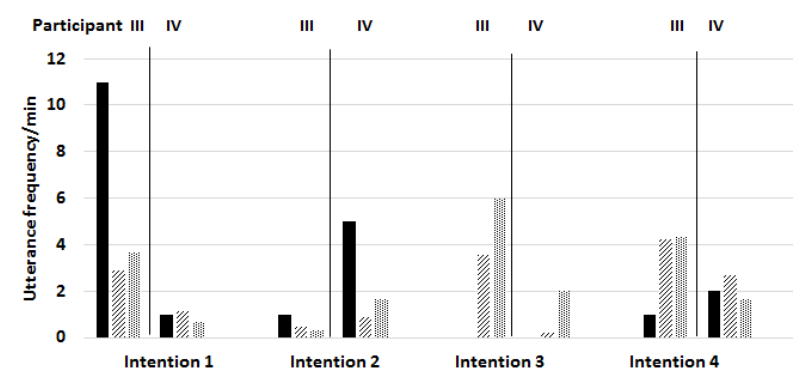

(b)

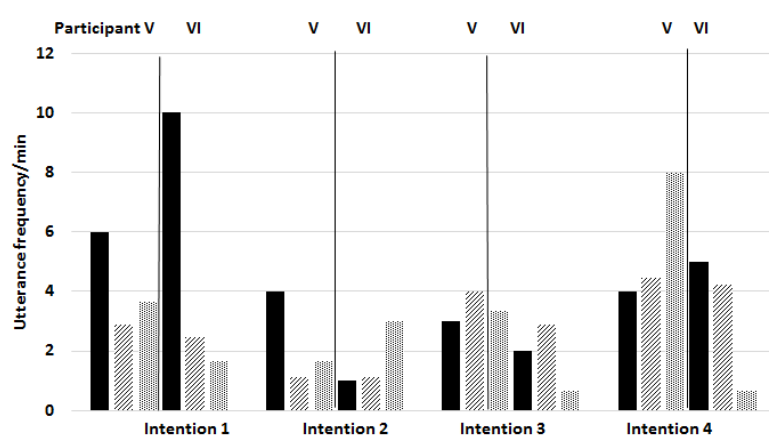

(c)

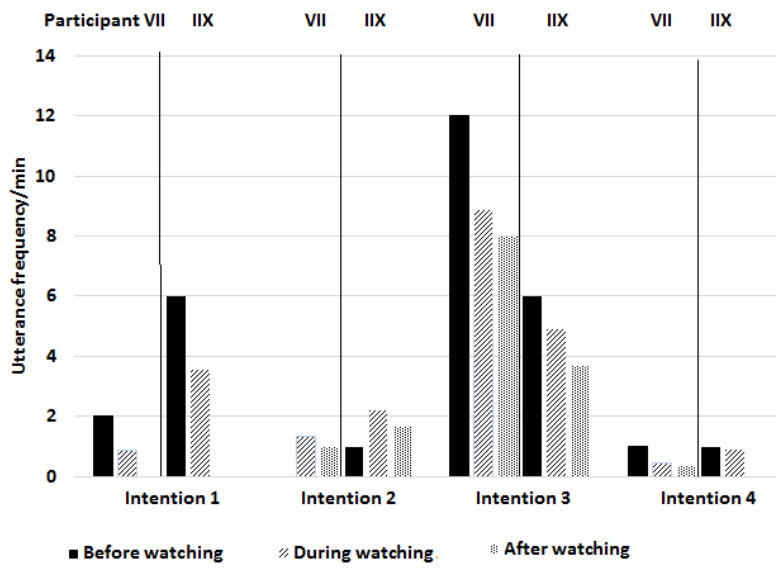

(d)

Fig.3 Time sequence changes of utterance intentions

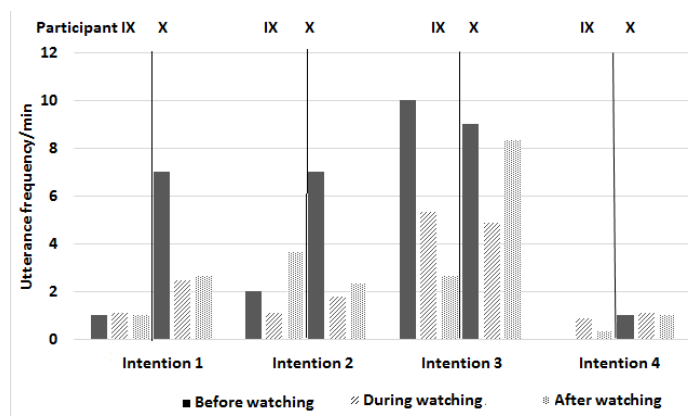

(e)

Fig.3 Time sequence changes of utterance intentions (continued)

\section{ACKNOWLEDGMENTS}

The authors express their sincere gratitude to the students who voluntary participated in this research.

\section{REFERENCES}

[1] S. G. Grand, C. Munchausen, P. Conrad, "Alternatives to Lecture: Experience Peer Instruction and Pedagogical Code Reviews", SIGCSE'14, March 5-8, 2014, Atlanta, Georgia, USA 15-64.

[2] L. Rodríguez-Vizzuett, J. L. Pérez-Medina, J. Muñoz-Arteaga, J. Guerrero-García, F. J. Álvarez-Rodríguez, 'Towards the Definition of a Framework for the Management of Interactive Collaborative Learning Applications for Preschoolers", Interacción '15, September 07 - 09, 2015

[3] J. Martens, F. Parthesius, B. Atasoy, "Design TeamMate : A Platform to Support Design Activities of Small Teams", AVI '10, May 25-29, 2010, Rome, Italy

[4] C. O'Malley, S. Langton, A. Andersont, G. Doherty-Sneddon and V. Bruce, "Comparison of face-to-face and video-mediated interaction", Interacting with Computers 018 no 2,177-192, 1996

[5] A. H. Anderson, A. Newlands, J. Mullin, A. M. Fleming, G. Sneddon and J. Van der Elden, "Impact of video-mediated communication on simulated service encounters", interacting with Computers $\sim 018$ no 2 , 193-206, 1996

[6] J. Carletta, A. H. Anderson, S. Garrod,'Seeing Eye to eye: an account of grounding and understanding in work groups", Cognitive studies: bulletin of the Japanese Cognitive Science Society, 9(1), 1-20, March 2002

[7] NHK Archives, NHK Creative Library, http://www.nhk.or.jp/archives/creative/material/ (April 23, 2017)

[8] DOVA-SYNDROME, FREE BGM, http://dova-s.jp/ (April 23, 2017)

[9] A.Shigeta, K. Hamamoto, and K. Nosu, "Estimation method of e-Learning learners' subjective difficulty by eye movement analysis of web-based English listening tests", Proceedings of E-Learn: World Conference on E-Learning in Corporate, Government, Healthcare, and Higher Education 2011, pp. 2516-2521, Oct. 2011, Honolulu, Hawaii, USA

[10] L. W. Anderson, D. R. Krathwohl, P. W. Airasian, K. A. Cruikshank, R. E. Mayer, P. R. Pintrich, J. Raths, M. C. Wittrock, ”A taxonomy for learning, teaching, and assessing: A Revision of Bloom's Taxonomy of Educational Objectives, Abridged Edition “, Pearson Education Limited; Pearson New International, 2013

[11] C. Dobson, Critical Thinking skills: Measuring higher cognitive development with Bloom's taxonomy, VDM Verlag, 2008 